\title{
SM Higgs searches at LHC
}

\author{
Luis R. Flores Castillo \\ University of Wisconsin-Madison
}

On behalf of the ATLAS and CMS collaborations

Hadron Collider Physics 2007

May $25^{\text {th }}, 2007$ 


\section{Outline}

- SM on Higgs

- Mass, production, decay

- LHC, detectors, performance

- Some channels

- Discovery potential

- Summary 


\section{SM Higgs mass constraints from data and theory}

\section{Experiment}

Indirect constraints from precision EW data :

$\mathrm{M}_{\mathrm{H}}<260 \mathrm{GeV}$ at $95 \% \mathrm{CL}$ (2004)

$\mathrm{M}_{\mathrm{H}}<186 \mathrm{GeV}$ with Run-I/II prelim. (2005)

$\mathrm{M}_{\mathrm{H}}<166 \mathrm{GeV}$ (2006, ICHEP06)

$\mathrm{M}_{\mathrm{H}}<153 \mathrm{GeV}$ (2007, CPT07)

2007: $\mathrm{M}_{H}<144 \mathrm{GeV}$, preliminary

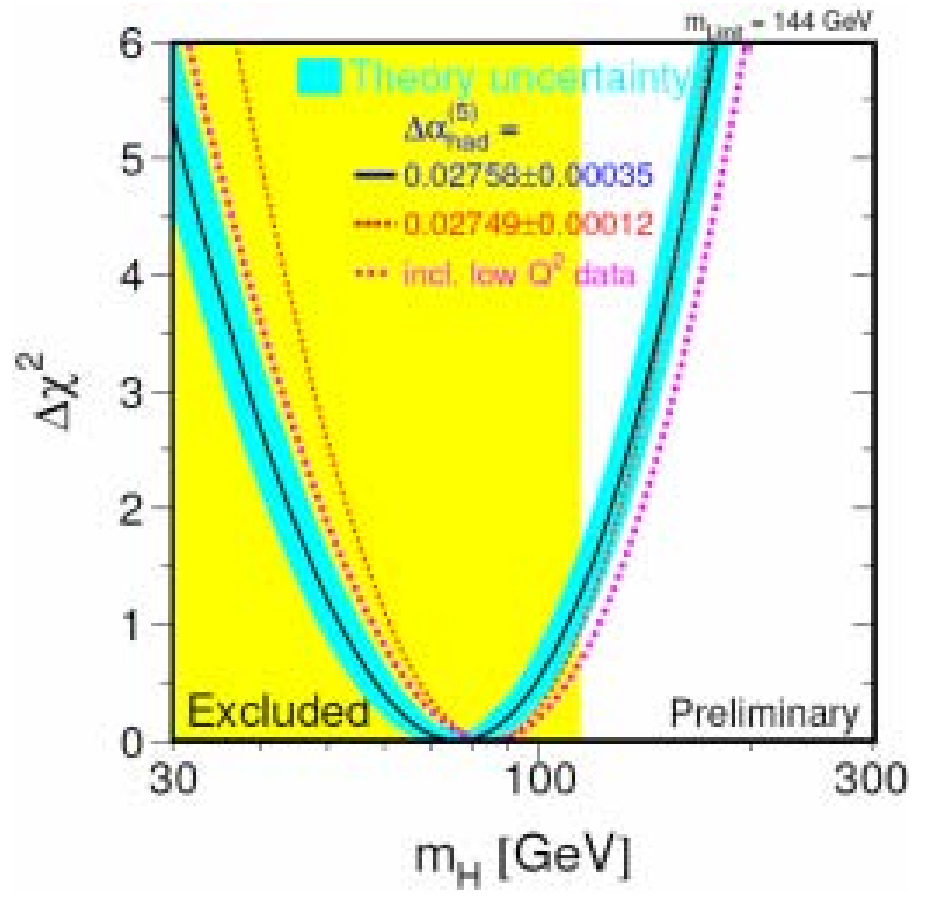

Direct limit from LEP: $M_{H}>114.4 \mathrm{GeV}$
SM theory

The triviality (upper) bound and vacuum stability (lower) bound as function of the cut-off scale $\Lambda$

(“Triviality" : finite Higgs self-coupling)

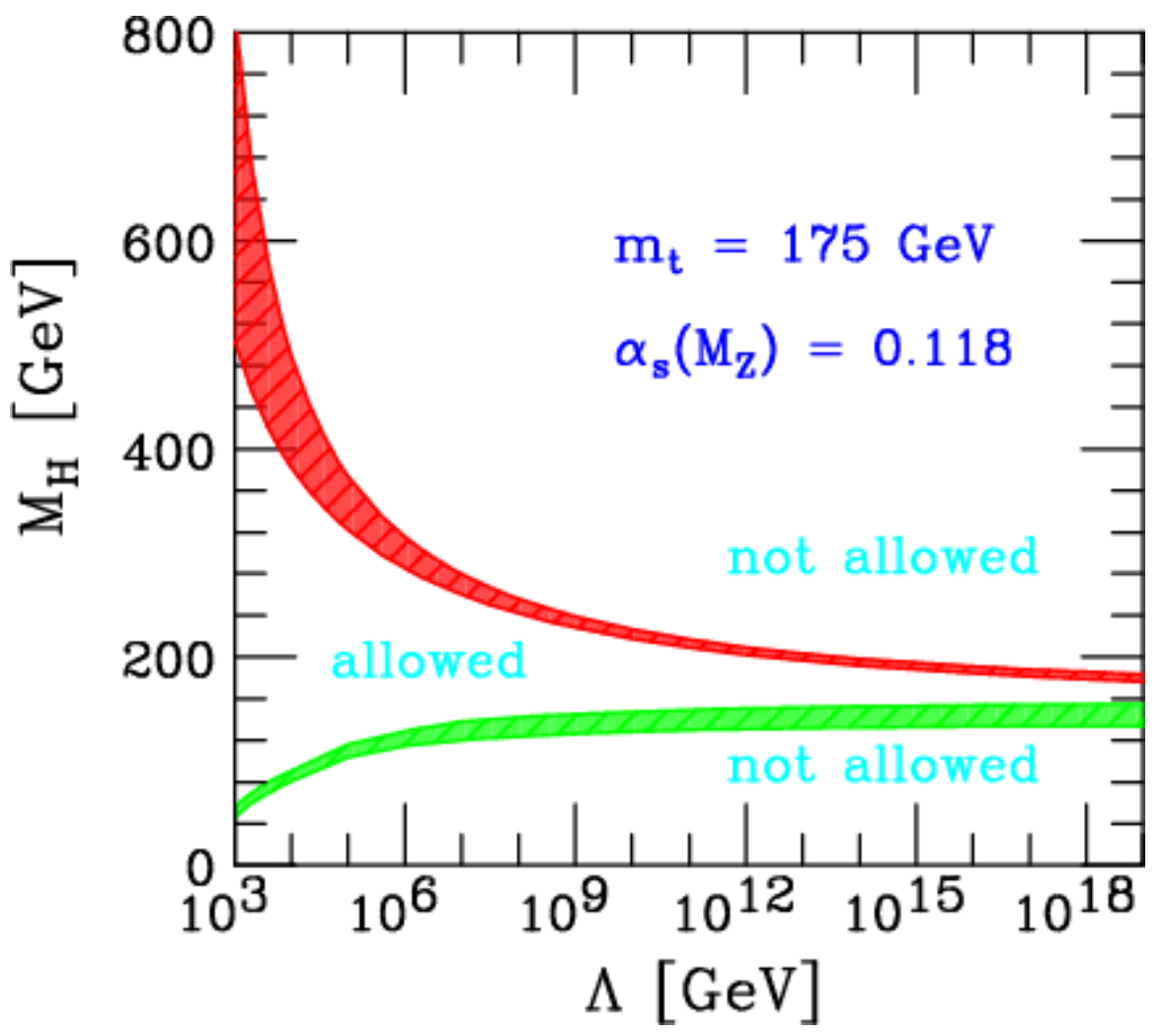




\section{SM Higgs: Production}
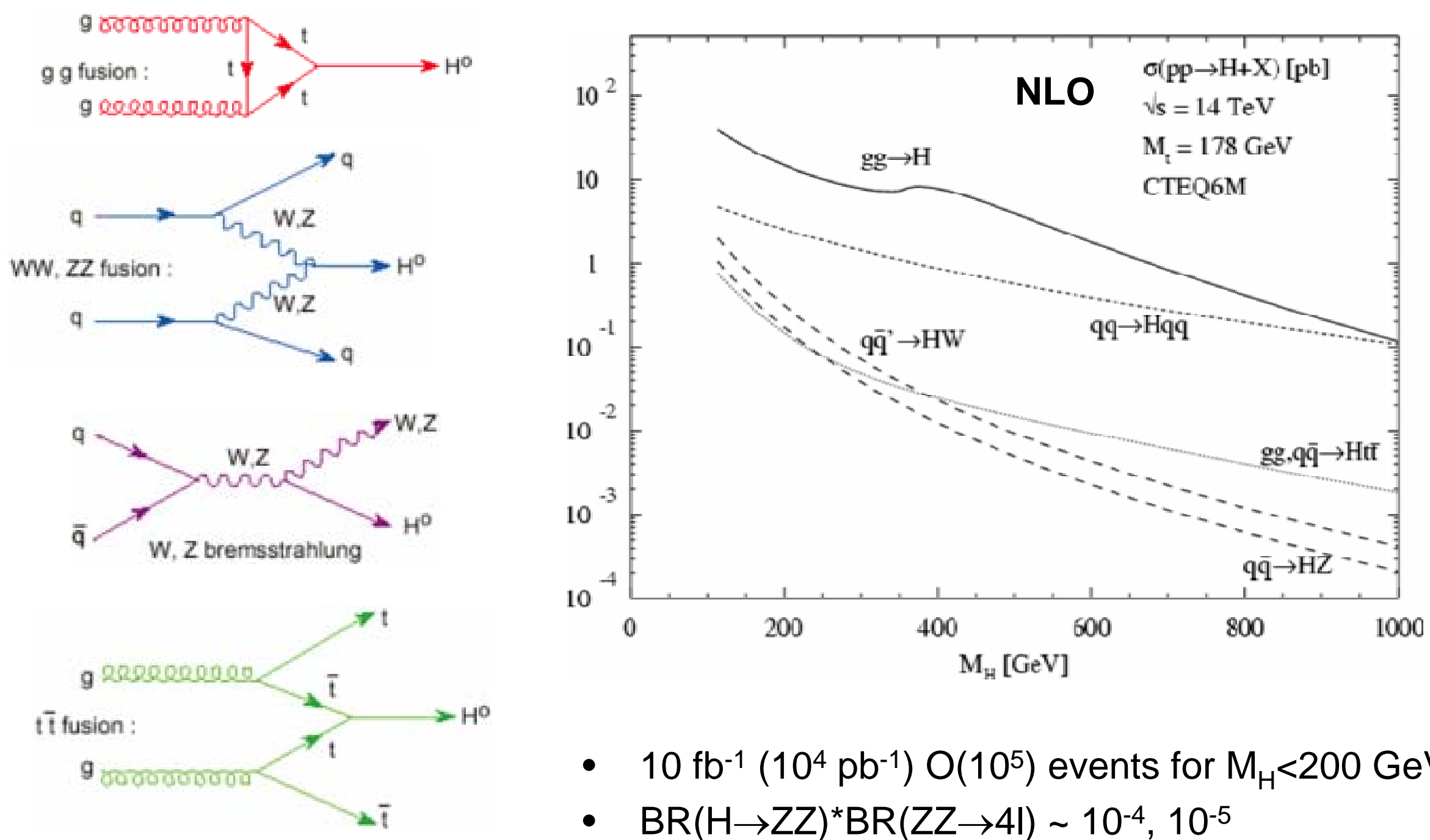

- $10 \mathrm{fb}^{-1}\left(10^{4} \mathrm{pb}^{-1}\right) \mathrm{O}\left(10^{5}\right)$ events for $\mathrm{M}_{\mathrm{H}}<200 \mathrm{GeV}$

- $\mathrm{BR}(\mathrm{H} \rightarrow \mathrm{ZZ})^{*} \mathrm{BR}(\mathrm{ZZ} \rightarrow 4 \mathrm{I}) \sim 10^{-4}, 10^{-5}$

- Few tens of $\mathrm{H} \rightarrow 4 \mathrm{l}$ events for $30 \mathrm{fb}^{-1}$ 


\section{SM Higgs: Decays}

\section{Djouadi, Kalinowski, Spira}
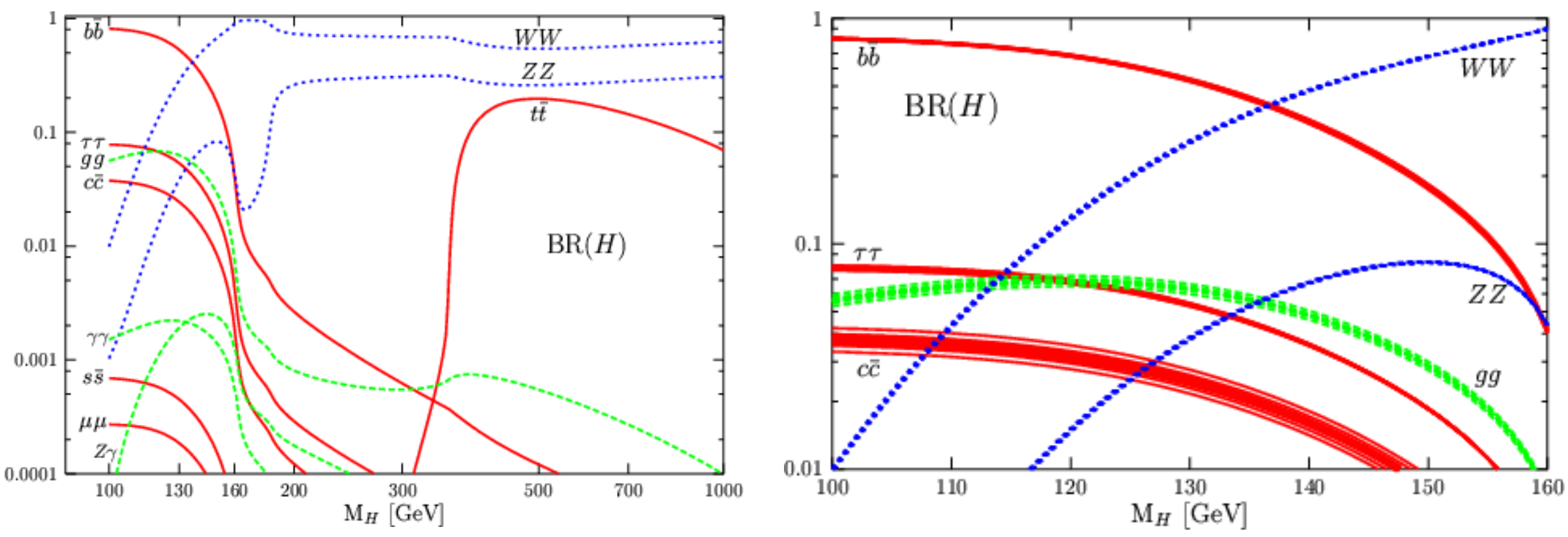

- Low mass $\left(\mathrm{M}_{\mathrm{H}}<2 \mathrm{M}_{\mathrm{Z}}\right)$ :

- bb dominant, but huge bb background $\rightarrow$ only $\mathrm{ttH}$ accessible

- Also accessible: $\mathrm{H} \rightarrow \tau \tau, \mathrm{H} \rightarrow Z^{*} \rightarrow 4 \mathrm{I}, \mathrm{H} \rightarrow \mathrm{WW}^{*} \rightarrow|v| v, \mathrm{H} \rightarrow \gamma \gamma$

- $\mathrm{M}_{\mathrm{H}}>2 \mathrm{M}_{\mathrm{Z}}$ :

- $\mathrm{H} \rightarrow \mathrm{ZZ} \rightarrow 4 \mathrm{I}$, WW modes

- Right plot: uncertainties from quark masses $(t, b, c)$ and $\alpha_{s}\left(M_{Z}\right)$ 


\section{Startup, environment}

- LHC startup

- All magnets in the tunnel; electrical, cryogenic and vacuum interconnections in progress

- Details: Philippe Lebrun's talk

- Detector construction well under way

- Commissioning: talks by Christoph Amelung and Pawel de Barbaro

- Physics environment

- pp@14 TeV

- "Low luminosity" phase: $\quad \sim 10^{33} \mathrm{~cm}^{-2} \mathrm{~s}^{-1} \rightarrow \sim 30 \mathrm{fb}^{-1}$

- "High luminosity": $\quad \sim 10^{34} \mathrm{~cm}^{-2} \mathrm{~s}^{-1} \rightarrow \sim 300 \mathrm{fb}^{-1}$

- Pile-up

- multiple pp interactions per bunch crossing (2 for low lumi, 20 for high lumi)

- on some detectors, information still in transit when new pp interaction occurs

- Triggers: from $40 \mathrm{MHz}$ (bunch crossing) to $\sim 200 \mathrm{~Hz}$ 


\section{Detectors}
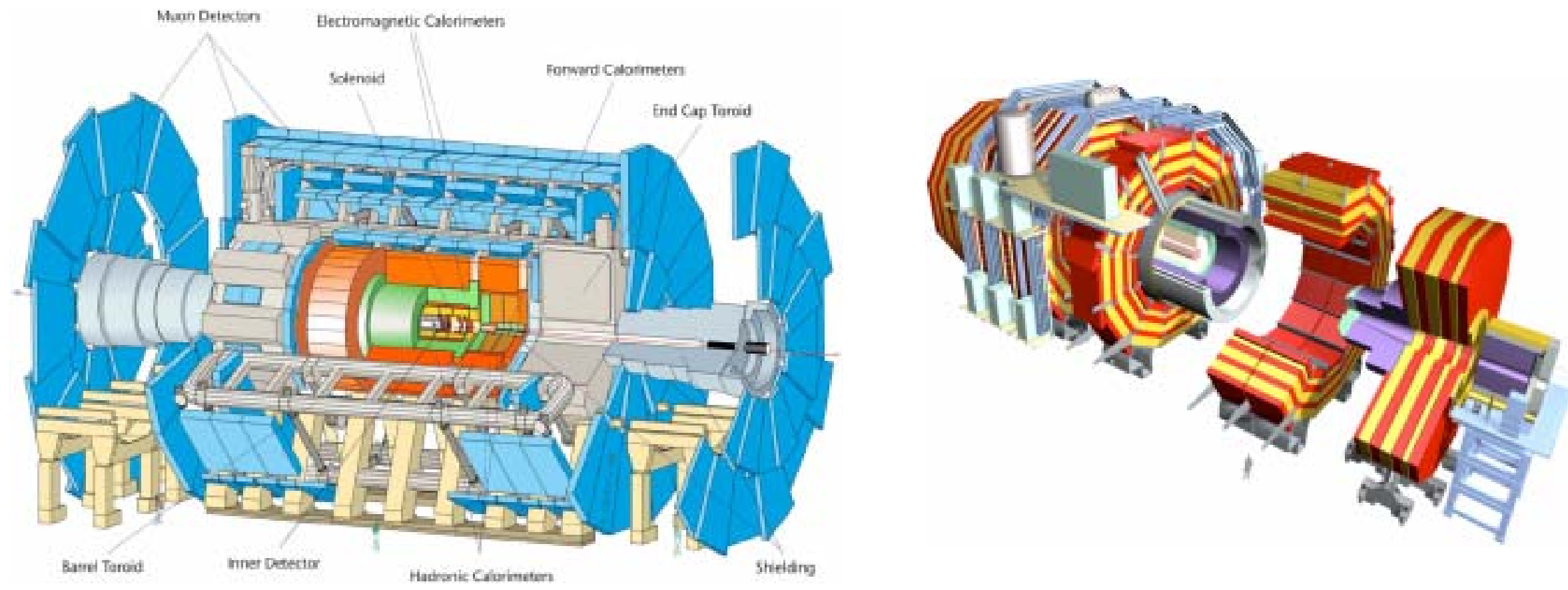

- Powerful id:

- Photons: Jet rejection $\sim$ few $10^{3}$ for $\sim 80 \%$ photon efficiency

- Electrons: Jet rejection $\sim 10^{5}$ for $\sim 80 \%$ electron efficiency

- B-jets: $\quad$ Light flavor jet rejection $\sim 100$ for $\sim 60 \%$ efficiency

- $\tau \rightarrow$ hadrons: Jet rejection $\sim$ few hundreds for $\sim 50 \%$ efficiency

- Energy resolution for e, $\gamma, \mu \sim 1-2 \%$

- Missing transverse momentum and jet reconstruction 


\section{Higgs Searches}

- "Benchmark" channels:

- Strongly tied to detector performance

- $\mathrm{H} \rightarrow \gamma \gamma, \mathrm{H} \rightarrow \mathrm{ZZ}^{(*)} \rightarrow 4 \mathrm{I}$

- Narrow peaks

- Event counting

- No peak

- Need good control of background normalization

- $\mathrm{H} \rightarrow \mathrm{WW}^{(*)}$

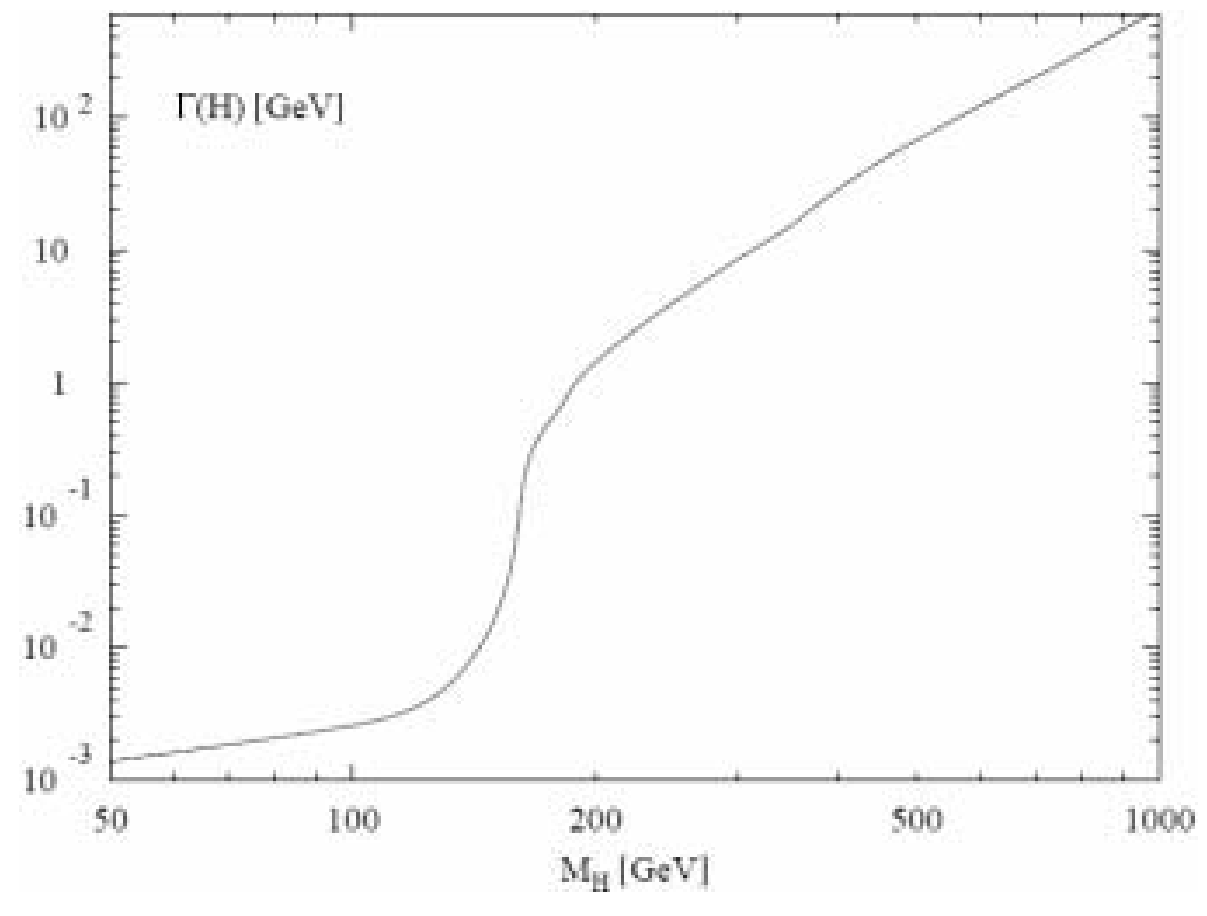

- VBF

- Take advantage of the special topology

- $\mathrm{H} \rightarrow \mathrm{WW}^{(*)}, \mathrm{H} \rightarrow \tau \tau$

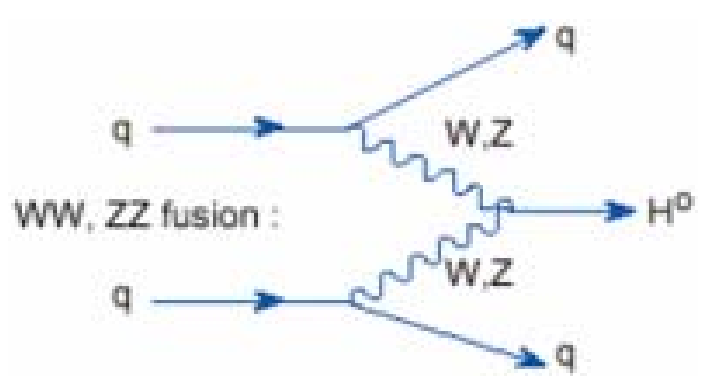




\section{$\mathrm{H} \rightarrow \gamma \gamma$}

- Narrow peak over smooth background

- Higgs' width negligible for low masses

- Relies on photon id, energy resolution and primary vertex determination

- Powerful at low $\mathrm{m}_{\mathrm{H}}\left(6\right.$ to $\left.8 \sigma @ 30 \mathrm{fb}^{-1}\right)$

- Photon id

- ATLAS: high granularity $\rightarrow$ high $\gamma / \pi^{0}$ separation

- Isolation cuts

- Recovery of conversions

- Different strengths, similar sensitivity

- ATLAS: photon id, angle

- CMS: energy resolution
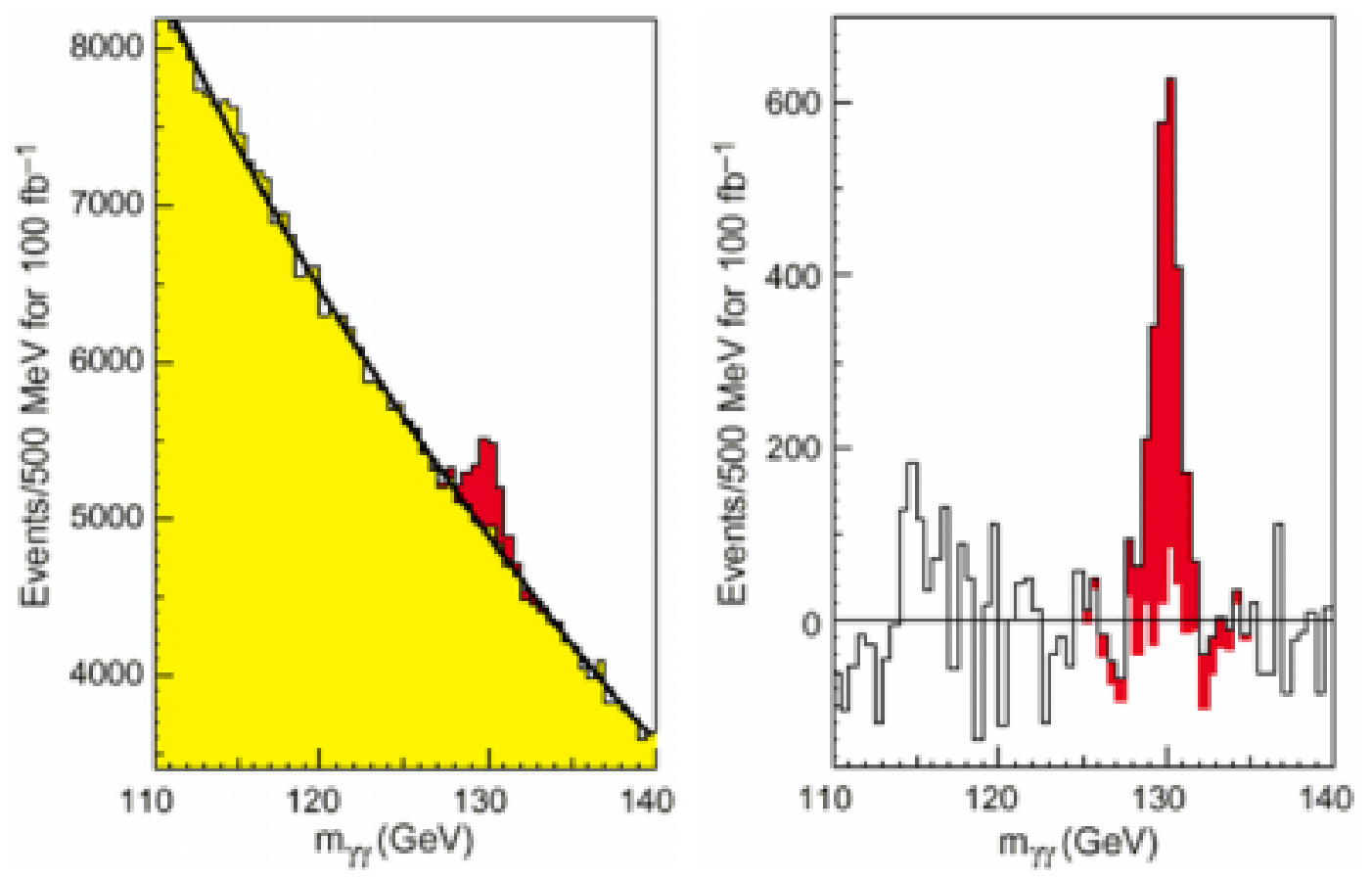


\section{$\mathrm{H} \rightarrow \gamma \gamma$}

\section{RECENT DEVELOPMENTS}

- Diphoton background now computed @ NLO

- agrees with Tevatron data

- Analysis improvements

- CMS "optimized analysis"

- Splitting into categories (depending on energy ratios and $\eta$ regions)

- Use of LLR for discovery, systematic

- NN with kinematics and $\gamma$ isolation as input, s/b per event

- ATLAS

- New discriminating variables, including Higgs $\mathrm{P}_{\mathrm{T}}$

- Higgs + jets

- Combined 0,1,2 jet analysis

- Classification of events

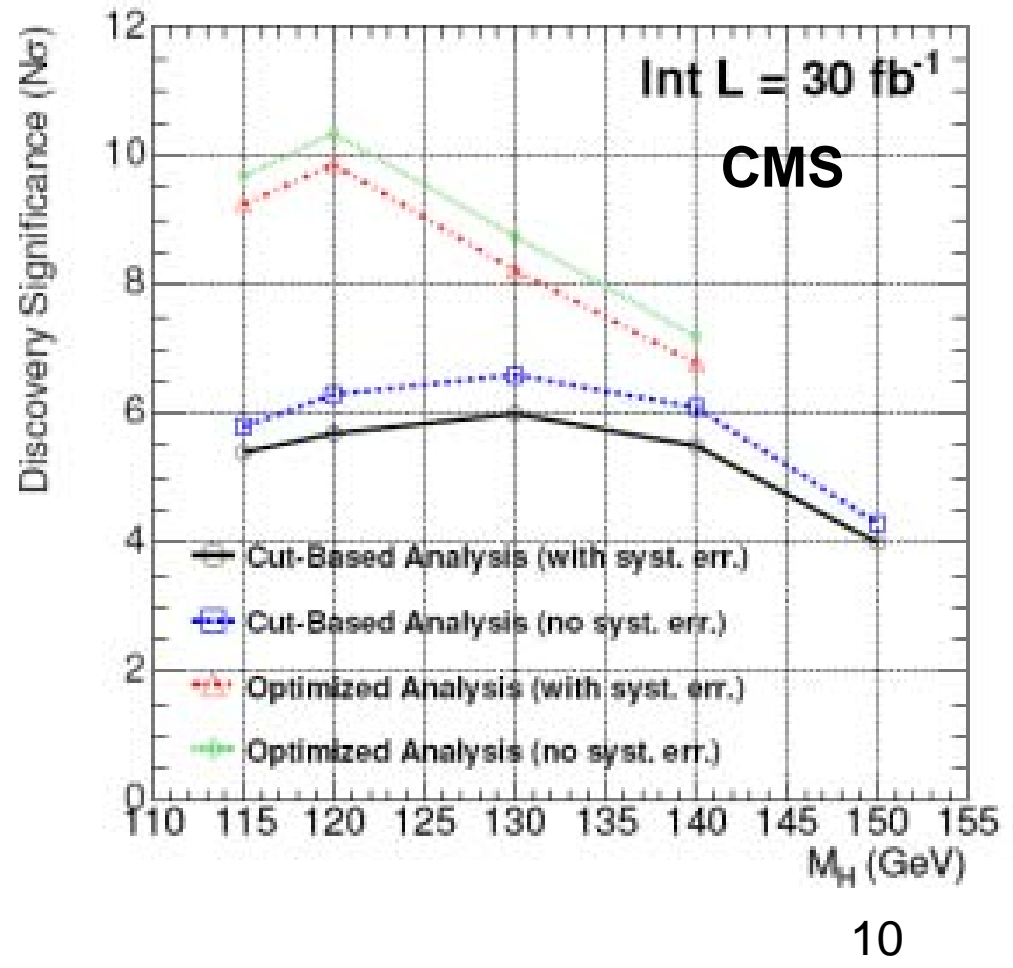




\section{$\mathrm{H} \rightarrow 4$ leptons}

- Relies on lepton id and energy resolution

- For $\mathrm{M}_{\mathrm{H}}=130 \mathrm{GeV}, 4 \mathrm{I}$ resolution (dominated by detector) 1.5 to $2 \mathrm{GeV}$

- Backgrounds

- ZZ ${ }^{(*)} \rightarrow 4 \mathrm{II}:$ qq $\rightarrow Z Z$ known @ NLO; 20\% added to account for $g g \rightarrow Z Z$

- $\mathrm{Zblb} \rightarrow 4 \|, \mathrm{t} \rightarrow \mathrm{t} \rightarrow \mathrm{l}:$ non isolated leptons, high impact parameter

- Need to understand lepton isolation and impact parameter significance

- Clean, but low statistics (especially for $M_{H}<130$ and $160 \mathrm{GeV}$ )
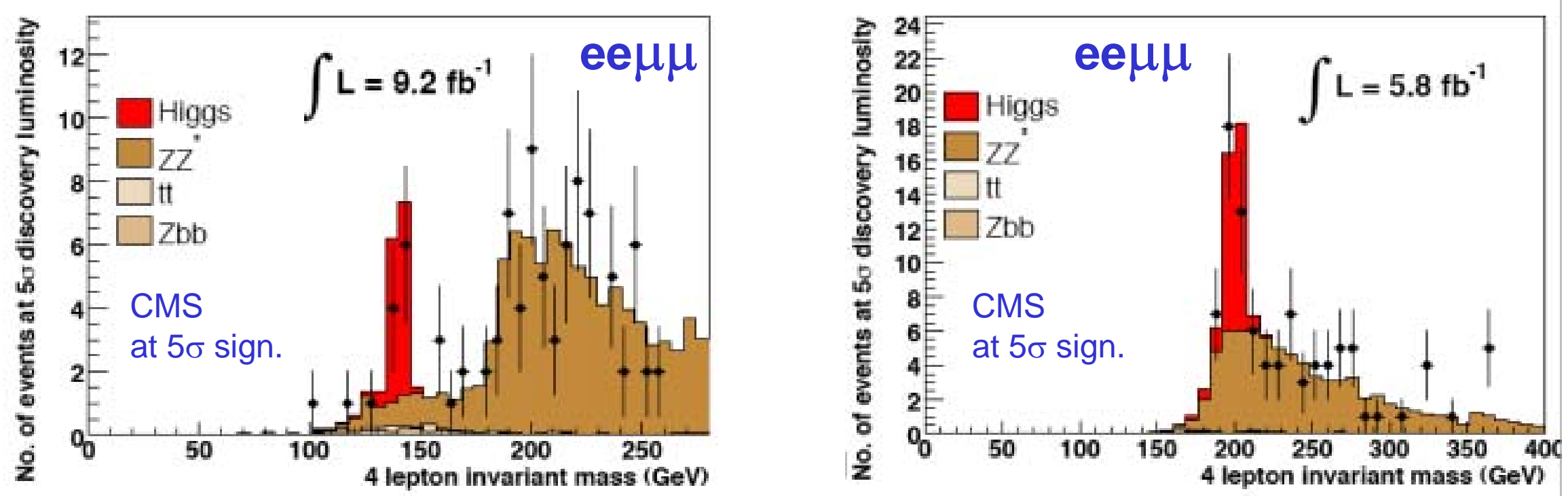

Poster session: Alexey Drozdetskiy (CMS) 


\section{$\mathrm{H} \rightarrow \mathrm{WW}^{(*)} \rightarrow \mathrm{lvlv}$}

- Event counting

- No mass peak

- Need accurate background estimate

- Control samples + extrapolation to signal region

- Most powerful around $\mathrm{M}_{\mathrm{H}} \sim 160 \mathrm{GeV}$

- $\mathrm{BR}(\mathrm{H} \rightarrow \mathrm{WW})>95 \%$

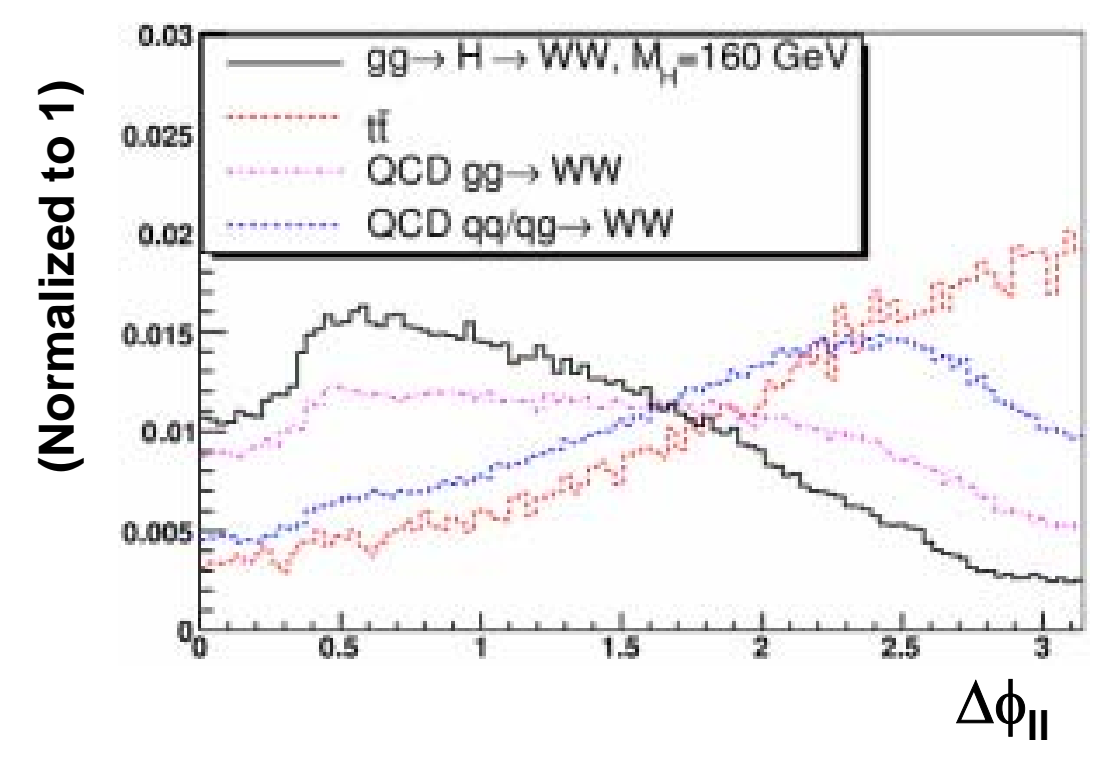

- Backgrounds

- tt: rejected by jet-veto

- WW: spin correlations

- Recently:

- $g g \rightarrow W W$ contribution

- tt, single top @NLO

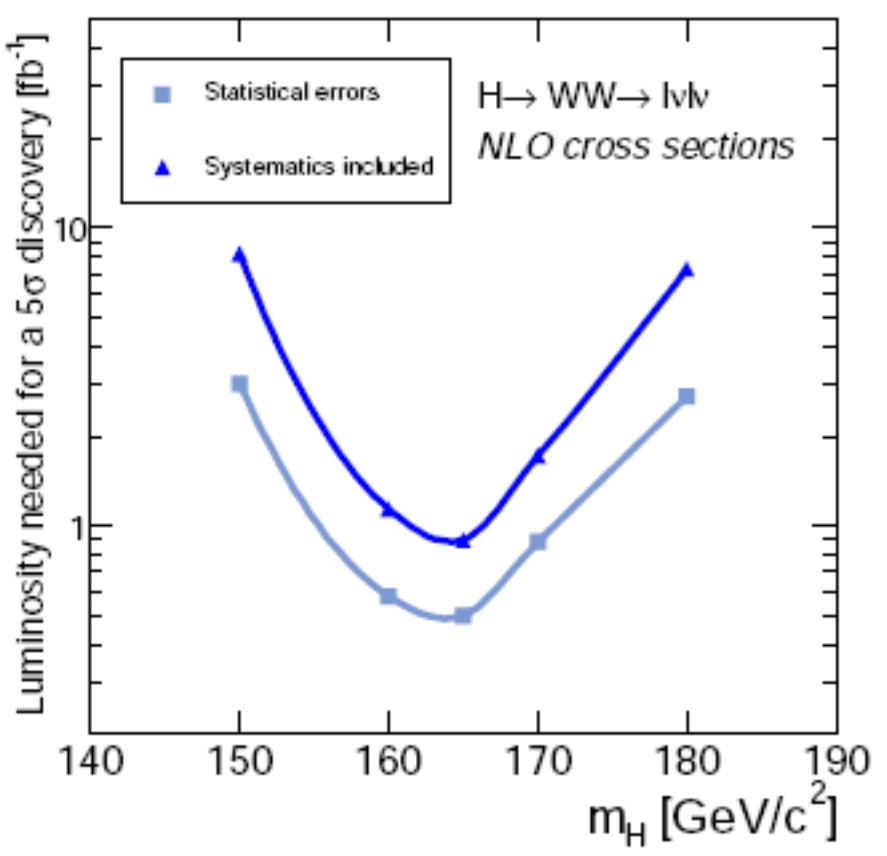




\section{SM Higgs and VBF}

D.Zeppenfeld, D.Rainwater, et al. proposed to search for a Low Mass Higgs in association with two jets with jet veto Central jet veto initially suggested in V.Barger, K.Cheung and T.Han in PRD 423052 (1990)
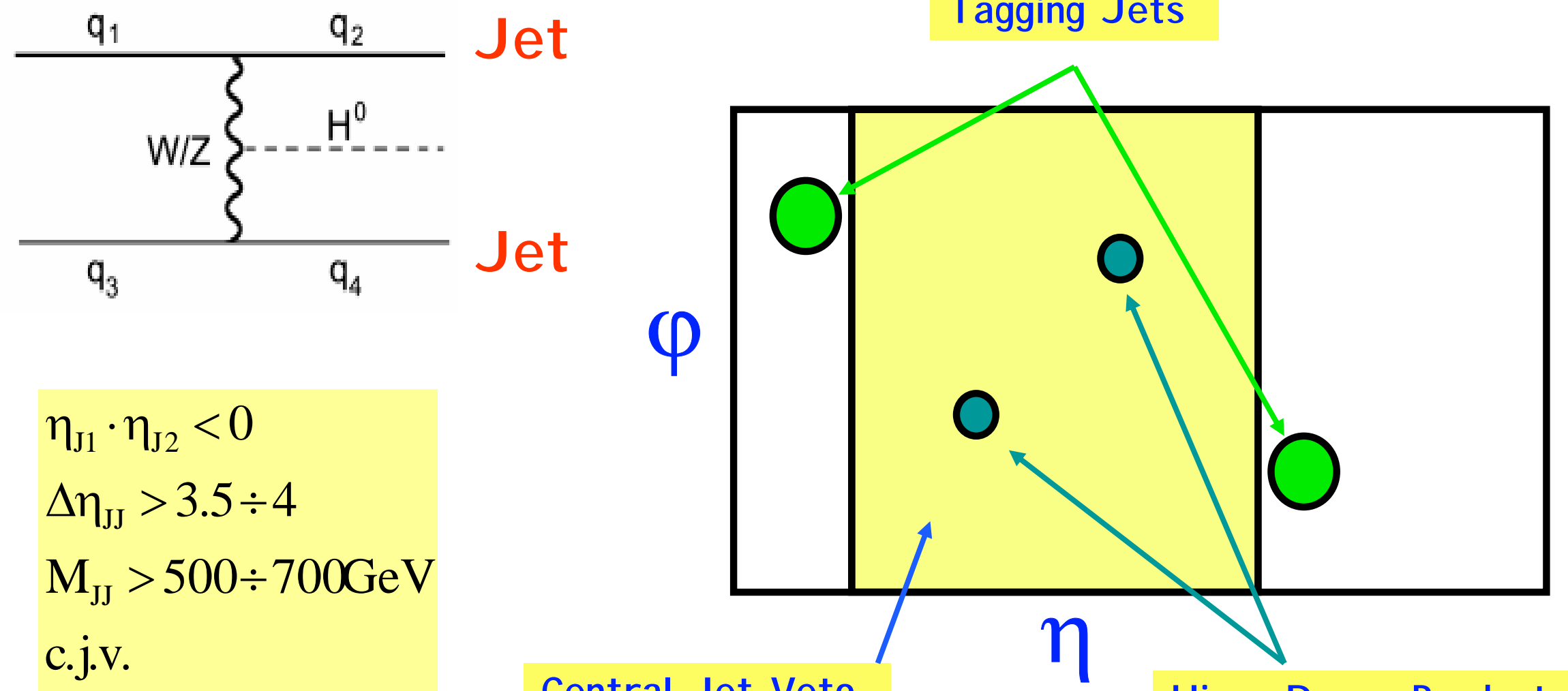

$$
\begin{aligned}
& \eta_{\mathrm{J} 1} \cdot \eta_{\mathrm{J} 2}<0 \\
& \Delta \eta_{\mathrm{JJ}}>3.5 \div 4 \\
& \mathrm{M}_{\mathrm{JJ}}>500 \div 700 \mathrm{GeV} \\
& \text { c.j.v. }
\end{aligned}
$$

Tagging gets

Higgs Decay Products 


\section{VBF $\mathrm{H} \rightarrow \tau \tau$}

- VBF selection:

- 2 "tagging" jets

- Higgs decay products between tagging jets

- Jet veto

- Final tau decay: Lepton-lepton or lepton-hadron

- Collinear approximation for mass reconstruction

- Resolution

- Limited by MET resolution (10 to $13 \mathrm{GeV}$ )

- Main background:

$-Z \rightarrow \tau \tau+2$ jets

- Most harmful at low $\mathrm{M}_{\mathrm{H}}$
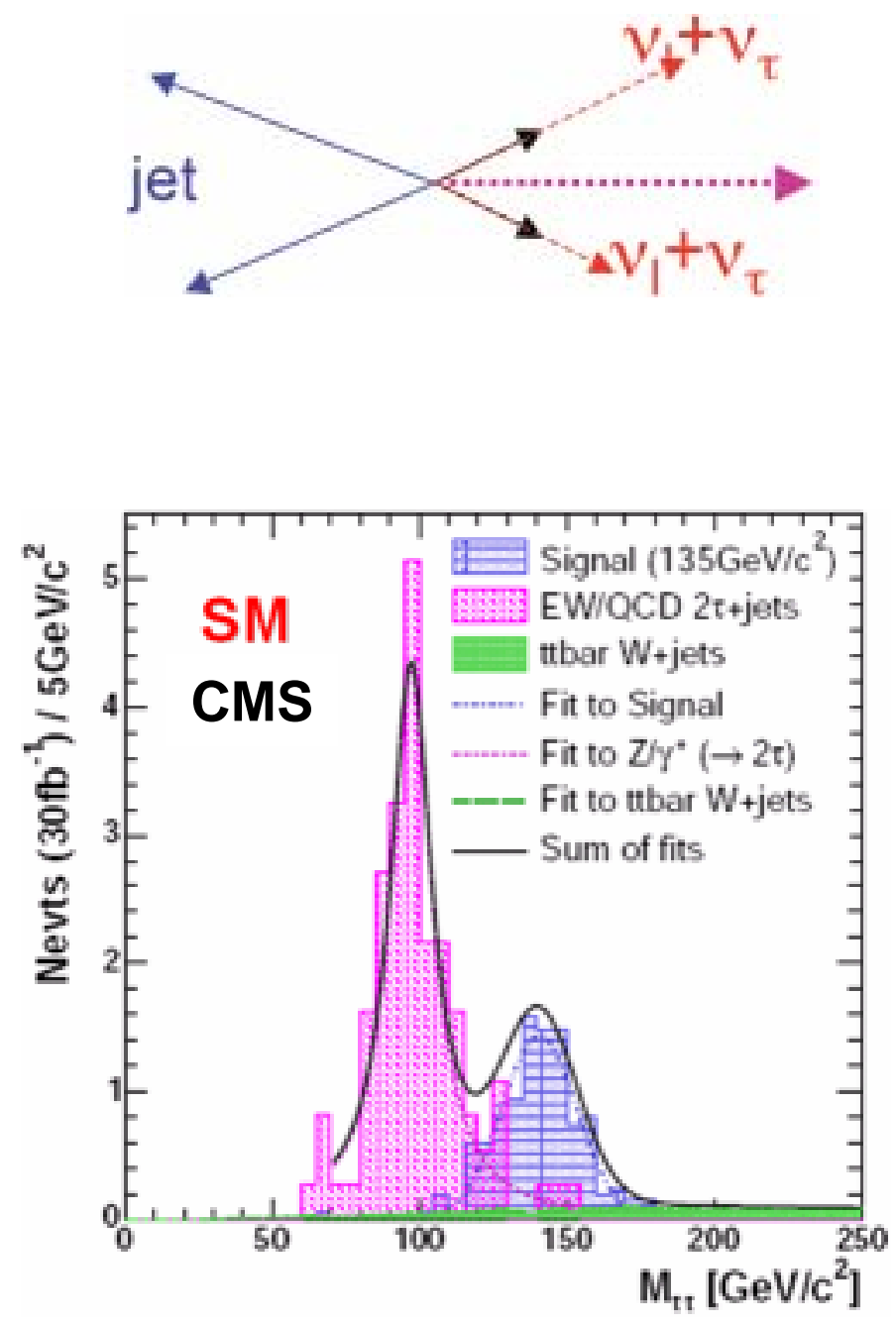


\section{Discovery potential $\left(30 \mathrm{fb}^{-1}\right)$}
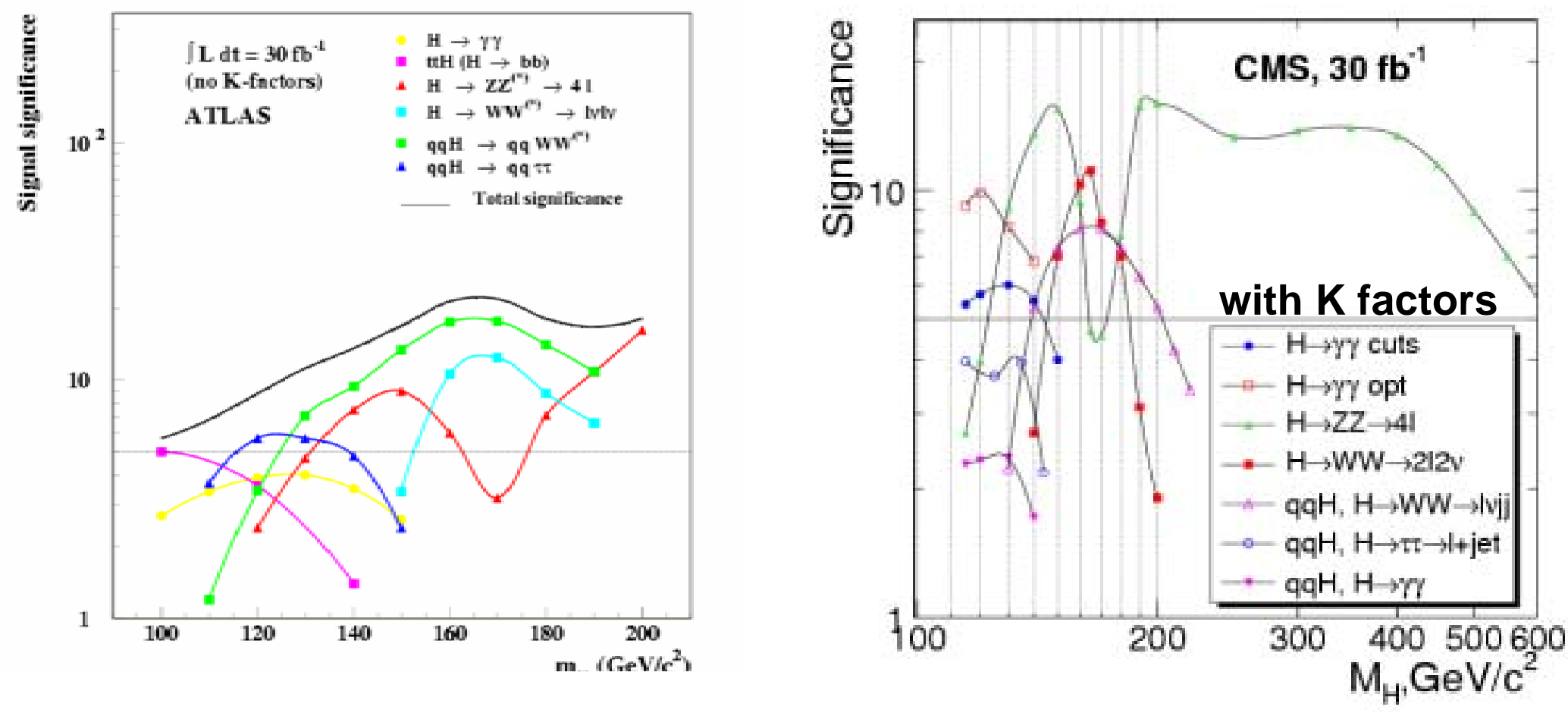

- Note the lack/use of $\mathrm{K}$ factors

- Several channels can be combined at low mass

- ATLAS $\mathrm{H} \rightarrow \gamma \gamma$ sensitivity is currently comparable with CMS

- Already good discovery potential with $10 \mathrm{fb}^{-1}$

- as long as detector performance and background systematics are under control 


\section{Summary, final comments}

- SM fits and experimental limits lead to a Higgs mass well within reach at $\mathrm{LHC}$

- Many SM Higgs channels have been studied in detail

- Already good sensitivity to SM Higgs with 10 fb-1

- Need to understand detector performance and background systematics early on

- Recent assessment of discovery potential at CMS; ATLAS is finalizing the last detailed studies before data taking

- Multivariate analyses in development along with cut based

- Strong attention paid to statistical methods to be used for the combination of different channels 
BACKUP 


\section{$\mathrm{ttH}(\mathrm{H} \rightarrow \mathrm{bb})$}
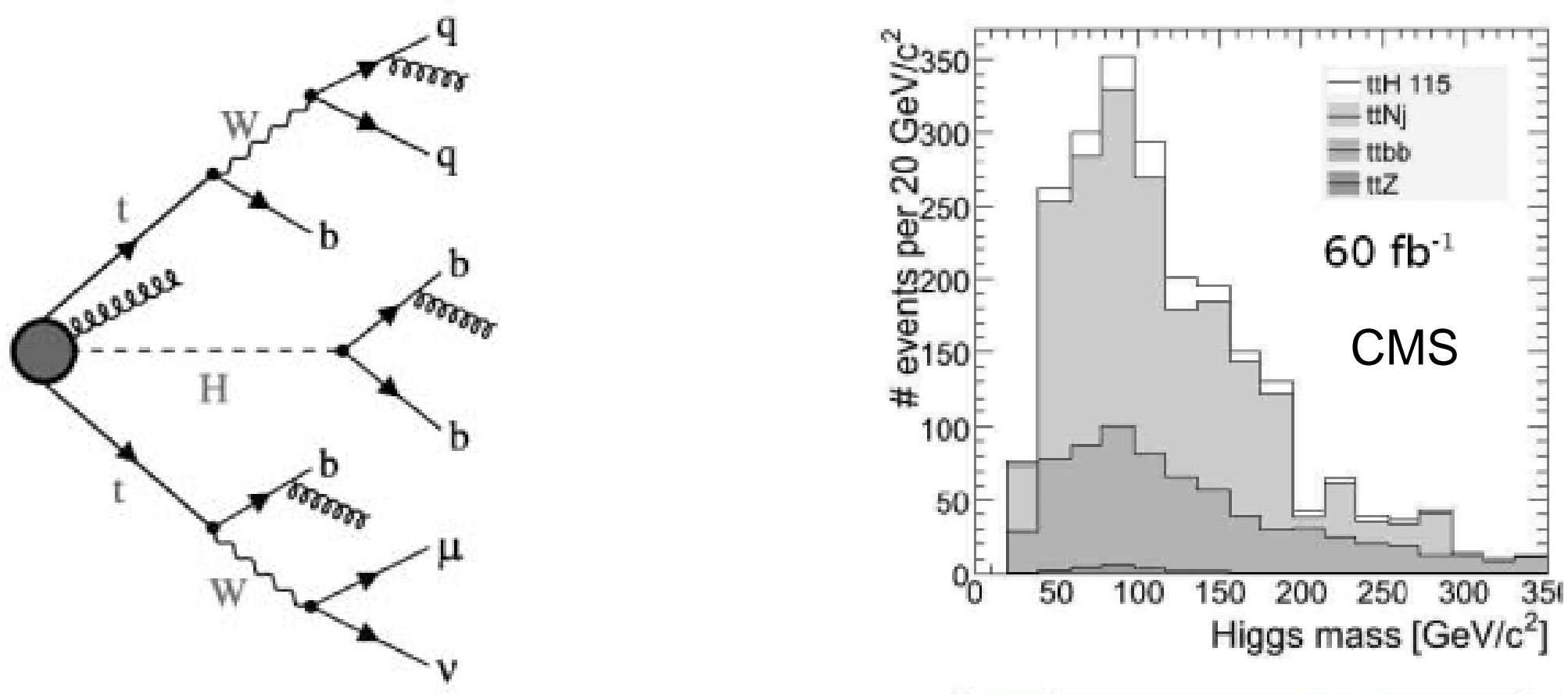

Latest CMS and ATLAS results are more pessimistic for this mode due to:

- effects of systematic included

- full detector simulation (b tagging, jet resolution)

- new $\mathrm{K}$ factors for signal

$\mathrm{ttH}, \mathrm{H} \rightarrow$ bb does not reach $3 \sigma$ at $60 \mathrm{fb}^{-1}$ with systematic included

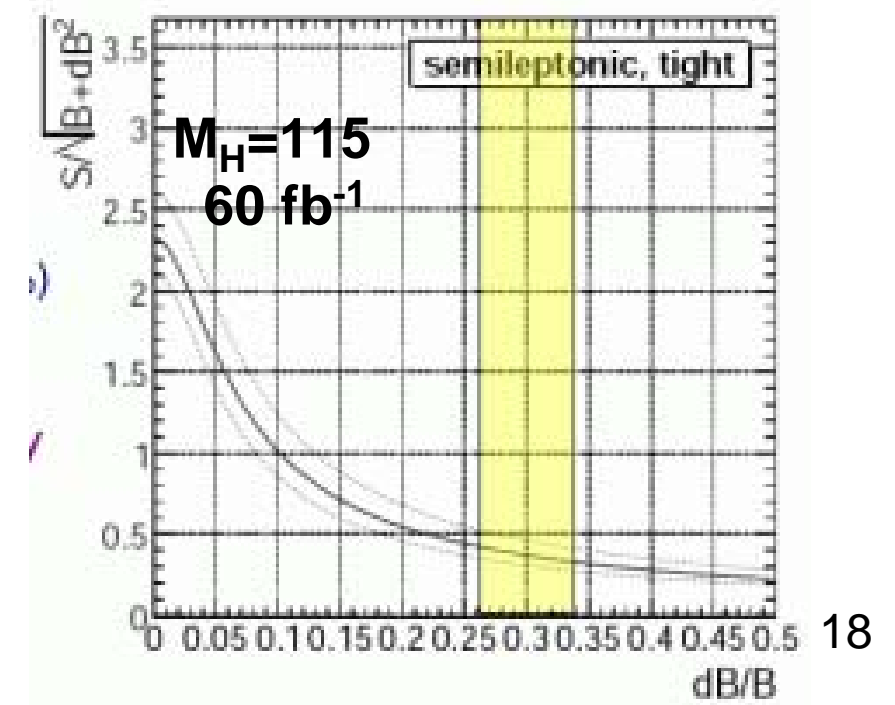

